\title{
TRAIL-induced caspase/p38 activation is responsible for the increased catalytic and invasive activities of Akt
}

\author{
BO K. SUN ${ }^{1 *}$, JOO-HANG KIM ${ }^{1,2 *}$, HOAN N. NGUYEN, SO Y. KIM ${ }^{1,2}$, \\ SEEUN OH ${ }^{1,2}$, YONG J. LEE ${ }^{3}$ and JAE J. SONG ${ }^{1}$ \\ ${ }^{1}$ Institute for Cancer Research, Yonsei Cancer Center; ${ }^{2}$ Brain Korea 21 Project for Medical Science, Yonsei University \\ College of Medicine, Seoul 120-752, Republic of Korea; ${ }^{3}$ Department of Surgery and Pharmacology, \\ School of Medicine, University of Pittsburgh, Pittsburgh, PA 15213, USA
}

Received August 2, 2010; Accepted September 16, 2010

\section{DOI: 10.3892/ijo_00000845}

\begin{abstract}
We previously observed that TRAIL induces acquired TRAIL resistance coinciding with increased Akt phosphorylation brought about by the Src-PI3K-Akt signaling pathways and mediated by c-Cbl. c-Cbl, a ubiquitously expressed cytoplasmic adaptor protein, is simultaneously involved in the rapid degradation of TRAIL receptors and Akt phosphorylation during TRAIL treatment. Here, we show that Akt phosphorylation is not exclusively responsible for acquired TRAIL resistance. Akt catalytic activation is known to increase during metabolic oxidative stress, but we show that TRAIL also dramatically induces the catalytic activation of Akt in TRAIL-sensitive cells, but not in TRAIL-resistant cells. This suggests that Akt catalytic activation during TRAIL-induced apoptosis is likely to play a compensatory role in the maintenance of cell homeostasis. In addition, activated p38 and phosphorylated HSP27 were found to act as downstream effector molecules of p38 during TRAIL treatment and were shown to be responsible for increased Akt catalytic and invasive activities.
\end{abstract}

Correspondence to: Dr Jae J. Song, Institute for Cancer Research, Yonsei Cancer Center, Yonsei University, 250 Seongsanno, Seodaemun-gu, Seoul 120-752, Republic of Korea

E-mail: jjs109@yuhs.ac

${ }^{*}$ Contributed equally

Abbreviations: TRAIL, tumor necrosis factor-related apoptosisinducing ligand; PAGE, polyacrylamide gel electrophoresis; c-Cbl, Casitas B-lineage lymphoma; FADD, Fas-associated death domain; PARP, poly (ADP-ribose) polymerase; PI3K, phosphoinositide-3 kinase; JIP1, JNK-interacting protein 1; MAPK, mitogen-activated protein kinase; JNK, c-Jun, NH2-terminal kinase; ERK, extracellular signal-regulated kinase; GSK-3, glycogen synthase kinase 3; HSP27, heat shock protein 27

Key words: TRAIL, p38, HSP27, Akt catalytic activity, invasion

\section{Introduction}

Tumor necrosis factor-related apoptosis-inducing ligand (TRAIL) is a potential anticancer agent. However, many cancer cell types, particularly some highly malignant tumors, are resistant to apoptosis induction by TRAIL. Furthermore, some cancer cells that were originally sensitive to TRAILinduced apoptosis can become resistant after repeated exposure (acquired resistance) $(1,2)$. Intrinsic resistance at the beginning of treatment can occur at different points in the signaling pathways of TRAIL-induced apoptosis (2). However, the mechanisms of acquired TRAIL resistance remain largely unknown for most cancer cells. TRAIL is likely to have other functions in addition to the induction of cell death. For example, it is known to stimulate the anti-apoptotic phosphoinositide-3 kinase (PI3K)/Akt pathway in endothelial cells (3) and fibroblasts (4) and to induce PI3K/Akt and nuclear factor- $\mathrm{B}(\mathrm{NF}-\kappa \mathrm{B})$ activation in Jurkat T leukemia cells (5). These findings imply that TRAIL can function as a cytokine of either cell death or cell survival depending on certain circumstances such as cellular context.

Trauzold et al (6) have reported that TRAIL promotes metastasis of human pancreatic ductal adenocarcinoma, demonstrating for the first time that TRAIL treatment strongly increases distant metastasis of pancreatic tumors in vivo. However, the molecular mechanism of TRAIL-induced tumor invasion and metastasis remains poorly understood. We previously noted that TRAIL can induce apoptosis of cancer cells when used as a chemotherapeutic drug. At the same time, TRAIL-resistant cells may develop the potential for progression to invasive or metastatic cancer by increasing pAkt and Bcl-xL expressions (7), which eventually lead to the degradation of the extracellular matrix, a typical sign of tumor invasion and metastasis (8). Human collagenase-3 (matrix metalloproteinase-13, MMP-13) belongs to the subfamily of interstitial collagenases that cleave stromal collagens types I and III (8). MMP-13 is a recently identified member of the MMP family that was originally isolated from breast carcinoma (9), and its wide substrate specificity also makes it a potent proteolytic tool for invading tumor cells (10-12). Monocyte chemotactic protein-1 (MCP-1) is a member of the chemokine superfamily that plays a key role in the recruitment and activation of monocytes during inflammation and angio- 
A

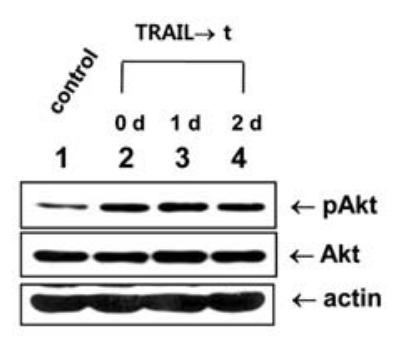

B

TRAIL $(200 \mathrm{ng} / \mathrm{ml})$

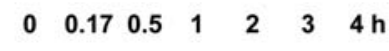

$\begin{array}{lllllll}1 & 2 & 3 & 4 & 5 & 6 & 7\end{array}$

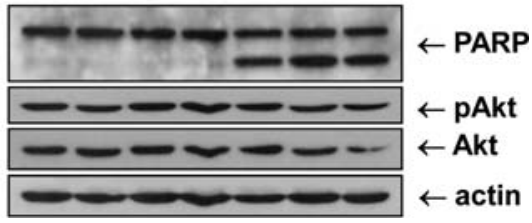

Figure 1. Akt phosphorylation during the development of acquired TRAIL resistance and during TRAIL treatment. (A) DU-145 cells were treated with TRAIL (200 ng/ml) for $4 \mathrm{~h}$. Detached cells were then removed by washing with PBS, followed by the addition of fresh media to the remaining attached cells, which were then incubated for the indicated times $(0,1,2$ days). Cells were lysed, and lysates were analyzed for phosphorylated Akt, and Akt. Actin was used to confirm equal amount of proteins loaded into each lane. Lane 1, control; lane 2, attached cells after 0 day of TRAIL; lane 3, attached cells after 1 day of TRAIL; lane 4 , attached cells after 2 days of TRAIL (B) DU-145 cells were treated with $200 \mathrm{ng} / \mathrm{ml}$ TRAIL for various times $(0-4 \mathrm{~h})$ Cells were lysed, and lysates were analyzed for the presence of PARP, phosphorylated Akt and Akt. Actin was used to confirm that equal amounts of protein were loaded into each lane.

genesis (13). Lu et al (14) have demonstrated that MCP-1 also promotes prostate cancer cell proliferation and invasion in vitro by correlating higher MCP-1 patient serum levels with various advanced stage cancers.

Previously, we observed that TRAIL increases Akt phosphorylation and induces the Akt signaling pathway through the involvements of Rous sarcoma oncogene cellular homolog (src), PI3K and pAkt via Casitas B-lineage lymphoma (c-Cbl) (15). However, we also found that the catalytic activity of Akt other than Akt phosphorylation was increased by the dissociation of its negative regulator JNK-interacting protein (JIP1), as a negative feedback against metabolic oxidative stress (16). We thus hypothesized that the downstream signaling pathways of TRAIL and metabolic oxidative stress are somewhat similar in that they both induce c-Jun NH2terminal kinase (JNK)/p38 activation $(16,17)$. In general, different mitogen-activated protein kinases (MAPKs) are members of separate modules and are regulated by distinct extracellular stimuli. For example, extracellular signalregulated kinases (ERKs) are activated by receptor tyrosine kinases and play a central role in mitogenic signaling $(18,19)$, while JNK and p38-type MAPKs are activated predominantly by stress stimuli and pathogenic insults (18). However, unlike their known typical patterns to corresponding stimuli, there are also many reports that each MAPK has pro- or antiapoptotic functions depending on cell type, nature of the death stimulus, duration of activation and the activities of other signaling pathways (20-23).
Activated $\mathrm{p} 38$ phosphorylates mitogen-activated protein kinase-activated protein kinase 2 (MAPKAPK2), which in turn phosphorylates HSP27 $(24,25)$. The mammalian small stress protein HSP27 is a phosphoprotein that forms large oligomers (up to $800 \mathrm{kD}$ ) with strong antiapoptotic properties that acts as a molecular chaperone to protect cells from heat shock and oxidative stress (26). Small HSPs (sHSPs; monomers of $15-42 \mathrm{kD}$ ) act as molecular chaperones to prevent unfolded proteins from irreversible aggregation, cooperating with other factors, e.g., HSP70 and ATP, to facilitate productive refolding (27). In addition, mammalian sHSPs are rapidly phosphorylated in response to various extracellular stresses, including oxidative stress, by MAPK-activated proteins kinase 2 , a substrate of p38 $(25,28)$. Increased phosphorylation of mammalian sHSPs leads to changes in oligomeric organization. For instance, induction of HSP27 phosphorylation leads to reduction of the oligomeric size to $\sim 70-250 \mathrm{kD}$ and is accompanied by the down-regulations of both chaperone action and resistance against oxidative stress (27). However, more recently, Xu et al (29) showed that HSP phosphorylation induced by p38 MAP kinase was necessary for the transforming growth factor- $\beta$ (TGF- $\beta$ )-mediated increase in MMP-2 and cell invasion in human prostate cancer. Here, we investigated the possibility of Akt catalytic activation in TRAIL-induced signaling pathways. We found that TRAIL-induced p38 activation and subsequent HSP27 phosphorylation were responsible for increased Akt catalytic and invasive activities.

\section{Materials and methods}

Cell culture. A human prostate adenocarcinoma cell line, DU-145, and SK-OV-3, a human ovarian carcinoma cell line, were cultured in Dulbecco's modified Eagle's medium (DMEM) with 10\% fetal bovine serum (FBS) (HyClone, Logan, UT, USA) and $26 \mathrm{mM}$ sodium bicarbonate. A human prostate adenocarcinoma cell line, $\mathrm{LNCaP}$, was cultured in RPMI-1640 with $10 \%$ FBS and $26 \mathrm{mM}$ sodium bicarbonate. The cells were maintained in a $37^{\circ} \mathrm{C}$ humidified atmosphere containing $5 \% \mathrm{CO}_{2}$ and air.

Reagents and antibodies. The p38 inhibitor SB203580 was purchased from Calbiochem (San Diego, CA, USA). AntiAkt, anti-Bad, anti-phospho-Bad, anti-GSK-3, anti-phosphoGSK-3, anti-p38, anti-phospho-p38, anti-phosphoS473-Akt and anti-Bcl-xL antibodies were purchased from Cell Signaling (Beverly, MA, USA). Anti-MMP13 antibody and protein G were purchased from Santa Cruz Biotechnology (Santa Cruz, CA, USA). Anti-actin antibody was purchased from ICN (Costa Mesa, CA, USA). Anti-HSP27 and anti-phospho-HSP27 (Ser 78) antibodies were purchased from Assay Designs (Ann Arbor, MI, USA). Monoclonal anti-PARP antibody was purchased from Biomol International, L.P. (Plymouth Meeting, PA, USA). Other chemicals were purchased from Sigma-Aldrich (St. Louis, MO, USA).

Protein extracts and polyacrylamide gel electrophoresis. Cells were lysed with 1X Laemmli lysis buffer (2\% sodium dodecyl sulfate, $10 \%$ glycerol, $0.002 \%$ bromophenol blue and $62.5 \mathrm{mM}$ Tris, $\mathrm{pH} \mathrm{6.8)} \mathrm{and} \mathrm{boiled} \mathrm{for} 10 \mathrm{~min}$. Protein 
A

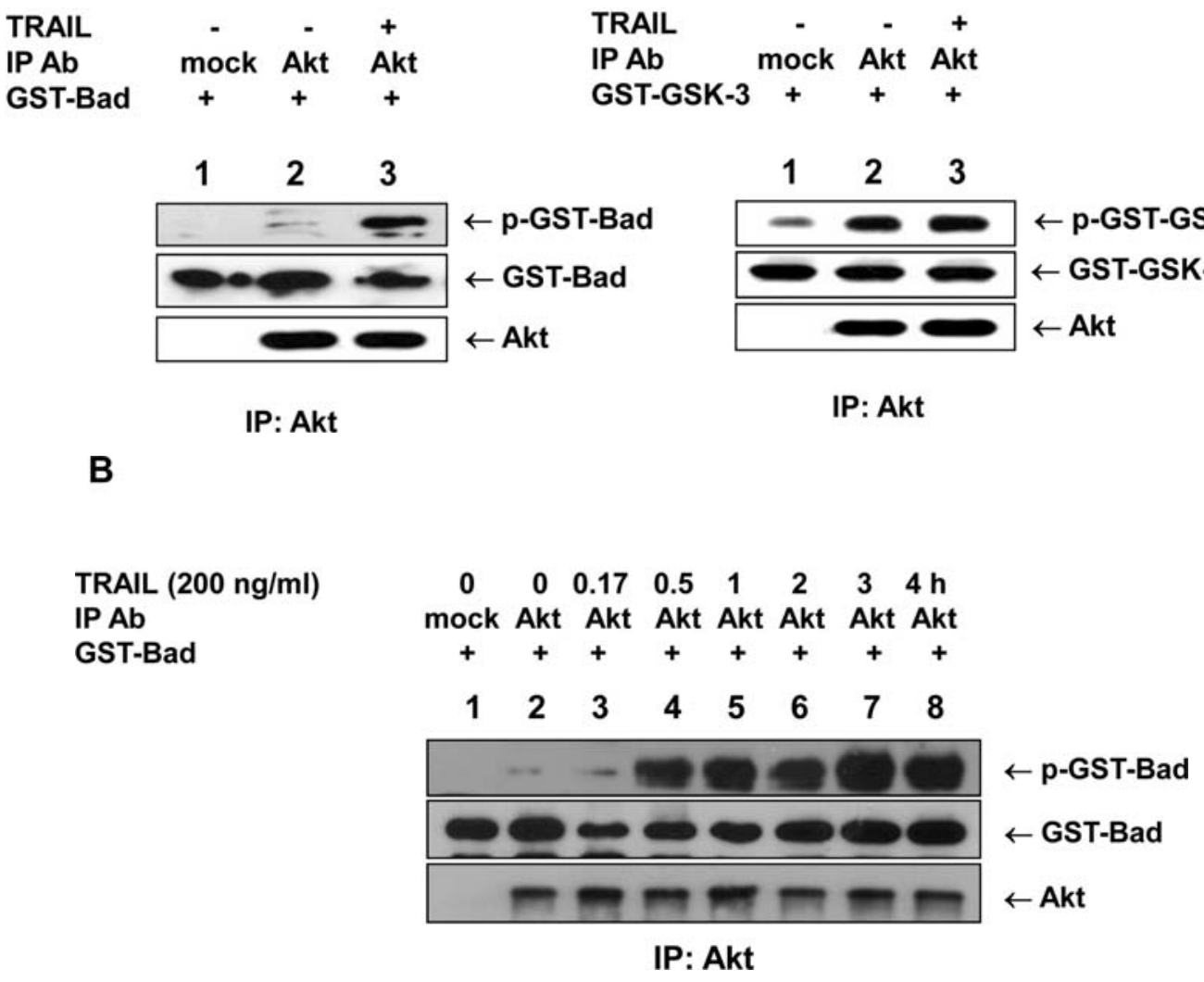

Figure 2. Akt catalytic activity during TRAIL treatment. (A) DU-145 cells were treated with $200 \mathrm{ng} / \mathrm{ml}$ TRAIL for 4 h. Cells were lysed, and lysates were immunoprecipitated (IP) with mouse anti-Akt antibody. Catalytic activity of immunoprecipitated Akt after TRAIL treatment was estimated for GST-Bad or GST-GSK-3 fusion protein substrates. GST-Bad, phosphorylated GST-Bad, GST-GSK-3 and phosphorylated GST-GSK-3 were detected with anti-Bad, antiphospho-Ser-136-Bad, anti-GSK-3 $\alpha$, or anti-phospho-GSK-3 $\alpha / 3$ antibodies, respectively. Immunoprecipitated Akt was detected with rabbit anti-Akt antibody. (B) DU-145 cells were lysed after exposure to TRAIL (200 ng/ml) for various times, and lysates were immunoprecipitated with anti-Akt antibody. Akt catalytic activity was examined in vitro using GST-Bad protein as a substrate. GST-Bad and phosphorylated GST-Bad were detected with anti-Bad and antiphospho-Ser-136-Bad antibodies, respectively. Immunoprecipitated Akt was detected with rabbit anti-Akt antibody.

content was measured with BCA Protein Assay Reagent (Pierce, Rockford, IL, USA). The samples were diluted with $1 \mathrm{X}$ lysis buffer and $B$-mercaptoethanol (final concentration $350 \mathrm{mM}$ ), and then equal amounts of protein were loaded onto 10 or $15 \%$ sodium dodecyl sulfate (SDS)-polyacrylamide gels. SDS-PAGE analysis was performed according to the method of Laemmli using a Hoefer gel apparatus.

Immunoblot analysis. Proteins were separated by SDS-PAGE and were electrophoretically transferred to nitrocellulose membranes. Each nitrocellulose membrane was blocked with $5 \%$ non-fat dry milk in PBS-Tween-20 $\left(0.1 \%\right.$, v/v) at $4^{\circ} \mathrm{C}$ overnight. The membrane was then incubated with primary antibody (diluted according to the manufacturer's instructions) for $2 \mathrm{~h}$. Horseradish peroxidase conjugated anti-rabbit or anti-mouse IgG was used as the secondary antibody. Immunoreactive proteins were visualized by chemiluminescence (ECL, Amersham, Arlington Heights, IL, USA).

Immune complex kinase assay. For the immune complex kinase assay, DU-145, LNCaP, or SK-OV-3 cells were lysed after TRAIL treatment in a buffer solution containing $20 \mathrm{mM}$ Tris-HCl, pH 7.5, 150 mM NaCl, 5 mM EGTA, 10 mM NaF,
$1 \%$ Triton X-100, 0.5\% deoxycholate, $2 \mathrm{mM}$ DTT, $1 \mathrm{mM}$ sodium orthovanadate, $1 \mathrm{mM}$ PMSF, and protein inhibitor cocktail solution (Sigma-Aldrich). Cell extracts were clarified by centrifugation, and the supernatants were immunoprecipitated with mouse anti-Akt antibody and protein G agarose (Santa Cruz Biotechnology, Inc.). The beads were washed twice with a solution containing $150 \mathrm{mM} \mathrm{NaCl}, 20 \mathrm{mM}$ Tris$\mathrm{HCl}, \mathrm{pH}$ 7.5, $5 \mathrm{mM}$ EGTA, $2 \mathrm{mM}$ DTT, $1 \mathrm{mM}$ sodium orthovanadate, $1 \mathrm{mM}$ PMSF, and protein inhibitor cocktail solution, washed once more with the kinase buffer solution, and subjected to kinase assays. To examine the catalytic activity of Akt, glutathione-S transferase (GST)-tagged Bad fusion protein (Santa Cruz Biotechnology, Inc.) and glycogen synthase kinase 3 (GSK-3) fusion protein (Cell Signaling) were used as substrates. Bad $(1 \mu \mathrm{g})$ or GSK-3 was incubated with immunoprecipitated Akt in kinase buffer containing $20 \mathrm{mM}$ Tris- $\mathrm{HCl}, \mathrm{pH} 7.5,20 \mathrm{mM} \mathrm{MgCl}_{2}, 1 \mathrm{mM}$ sodium orthovanadate, $2 \mathrm{mM} \mathrm{DTT}$, and $20 \mu \mathrm{M}$ ATP at $30^{\circ} \mathrm{C}$ for $1 \mathrm{~h}$. The reaction was stopped by adding $2 \mathrm{X}$ Laemmli buffer. Phosphorylated proteins were resolved using SDS-PAGE and analyzed using immunoblotting with anti-phospho-Bad (Ser-136) or anti-phospho-GSK-3 $\alpha / \beta$ antibodies (Cell Signaling). 
A

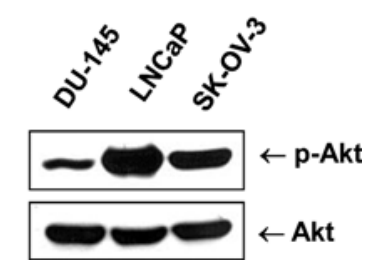

B
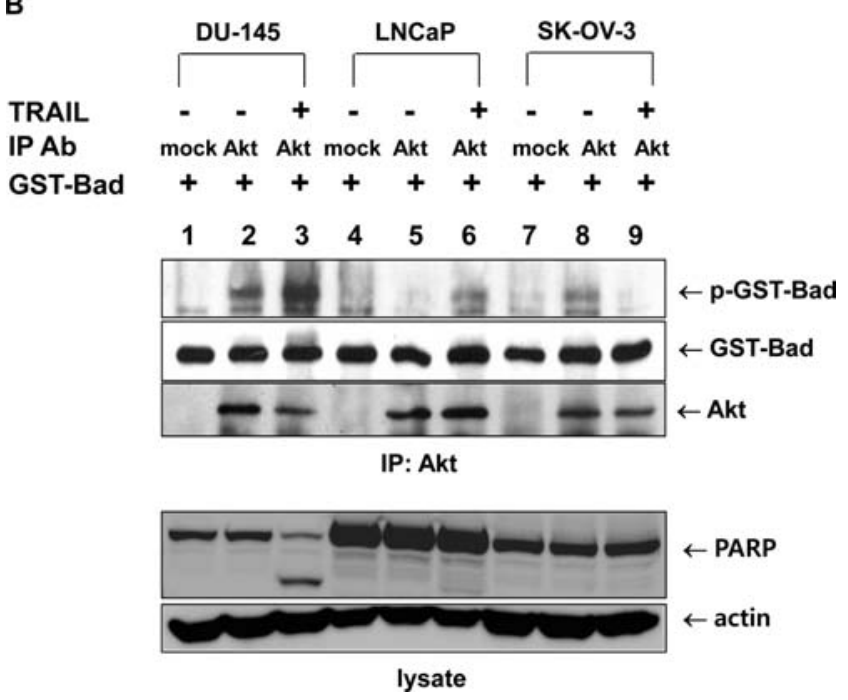

C

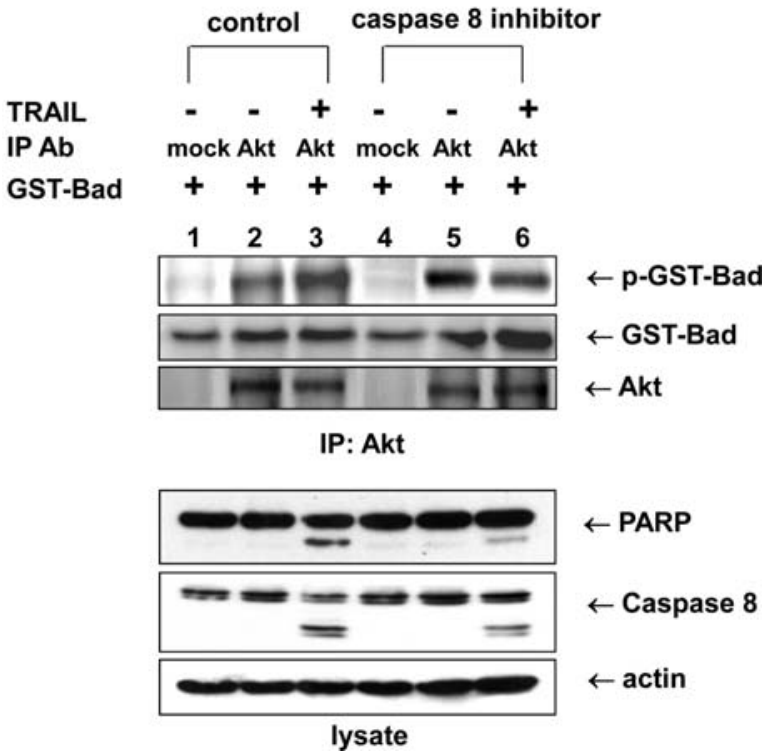

Figure 3. Akt catalytic activity dependence on TRAIL-sensitivity and the caspase 8-dependent signaling pathway. (A) Akt phosphorylation was estimated in TRAIL-sensitive DU-145 cells and TRAIL-resistant LNCaP and SK-OV-3 cells. Each cell line was treated with $200 \mathrm{ng} / \mathrm{ml}$ TRAIL for $4 \mathrm{~h}$, and lysates were immunoblotted with anti-phospho Akt antibody. (B) DU-145, LNCap and SK-OV3 cells were lysed, and the lysates were immunoprecipitated with mouse anti-Akt antibody. Akt catalytic activity was examined in vitro using GST-Bad protein as a substrate. GST-Bad and phosphorylated GST-Bad were detected with anti-Bad and anti-phospho-Ser-136-Bad antibodies, respectively. Immunoprecipitated Akt was detected with rabbit antiAkt antibody (upper panel). Cell lysates were immunoblotted with anti-PARP or anti-actin antibody, respectively (lower panel). (C) DU-145 cells were pretreated with caspase 8 inhibitor (Z-IETD-FMK $20 \mu \mathrm{M}, 30 \mathrm{~min}$ ), followed by TRAIL treatment $(200 \mathrm{ng} / \mathrm{ml})$ for $2 \mathrm{~h}$, and were lysed, and the lysates were immunoprecipitated with mouse anti-Akt antibody. Akt catalytic activity was examined in vitro using GST-Bad protein as a substrate. GST-Bad and phosphorylated GST-Bad were detected with anti-Bad and anti-phospho-Ser136-Bad antibodies, respectively. Immunoprecipitated Akt was detected with rabbit anti-Akt antibody (upper panel). Cell lysates were immunoblotted with anti-PARP, anti-caspase 8 or anti-actin antibody, respectively (lower panel).
Invasion assay. DU-145 cells were cultured in invasion chambers (Millipore, ECM551) after TRAIL treatment $(200 \mathrm{ng} / \mathrm{ml}$ for $4 \mathrm{~h})$ with or without SB203580 $(20 \mu \mathrm{M})$ pretreatment. DU-145 cells with acquired TRAIL resistance were cultured in invasion chambers with a thin layer of ECMatrix in serum-free media for $48 \mathrm{~h}$. Invasive cells that migrated through the ECM layer and adhered to the bottom of the polycarbonate membrane in $10 \%$ serum-containing media were stained.

MCP-1 ELISA assay. MCP-1 was quantified using a commercially available sandwich-type ELISA (R\&D Systems). DU145 cells pretreated with or without p38 inhibitor (SB203580 at $20 \mu \mathrm{M}$ for $1 \mathrm{~h})$ were then treated with TRAIL $(200 \mathrm{ng} / \mathrm{ml}$ for $4 \mathrm{~h}$ ). Cells were subsequently incubated with or without SB203580 for two days. Supernatants were harvested from attached DU-145 cells two days after TRAIL treatment and were incubated in wells pre-coated with a monoclonal antibody specific for MCP-1. After washing away any unbound substances, an enzyme-linked polyclonal antibody specific for MCP-1 was added to the wells. After washing, a substrate solution for color development was finally added to the wells, and the intensity of the color was measured for MCP-1 quantification.

\section{Results}

TRAIL induced Akt catalytic activity during apoptosis. We previously found that TRAIL-induced Akt phosphorylation plays an important role in the development of acquired TRAIL resistance (7), suggesting that TRAIL, in addition to being a death-inducing ligand, may also be involved in the survival of cancer cells (15). Here, we observed an increase in Akt phosphorylation in the attached cells after TRAIL treatment (Fig. 1A). However, Akt phosphorylation was harder to detect for TRAIL treatment at a concentration of $200 \mathrm{ng} / \mathrm{ml}$ (Fig. 1B) compared to those at lower concentrations (15). We questioned whether TRAIL was contributing to Akt activation only by increasing Akt phosphorylation or whether it was activating Akt in other ways to affect cell survival and acquired TRAIL resistance. We therefore examined whether Akt was being catalytically activated during TRAIL treatment as it was being catalytically activated under conditions of glucose deprivation (16). Surprisingly, Akt catalytic activity significantly increased during TRAIL treatment when Bad protein was used as an in vitro Akt substrate, but did not increase when GSK protein was used as a substrate (Fig. 2A). The increase in Akt catalytic activity began after $30 \mathrm{~min}$ of treatment and was sustained for $>4 \mathrm{~h}$ (Fig. 2B).

TRAIL-induced Akt catalytic activation is dependent on the activated caspase 8-dependent phospho-p38/phospho-HSP27 signaling pathways. Next, we investigated the reason for the increased Akt catalytic activity during TRAIL treatment. We hypothesized that the catalytic activation of Akt was a compensatory mechanism for protection of the cell from TRAIL-induced apoptosis. We therefore investigated whether catalytic activation of Akt also occurred in TRAIL-resistant cell lines such as LNCaP (30) or SK-OV-3 (31), which have 
A

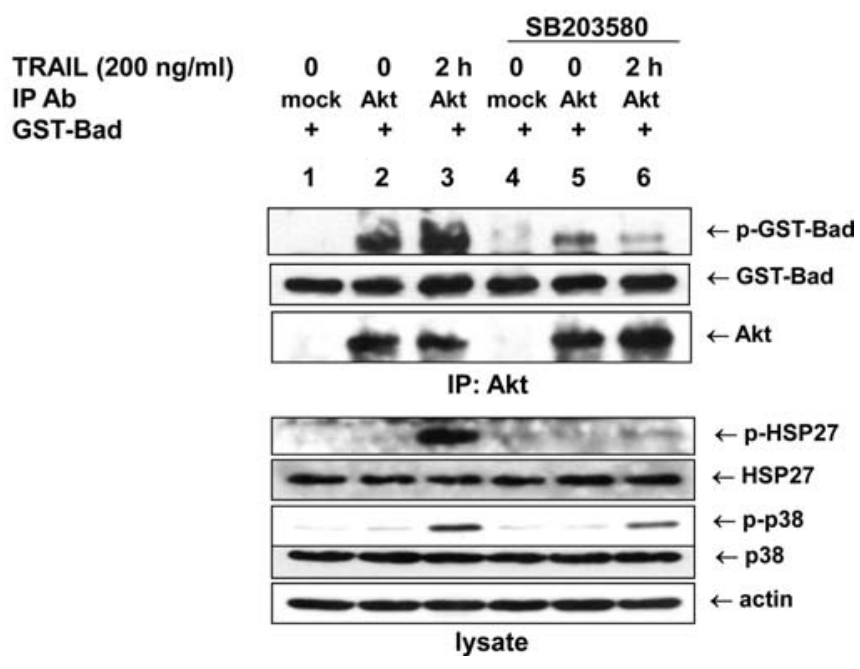

B

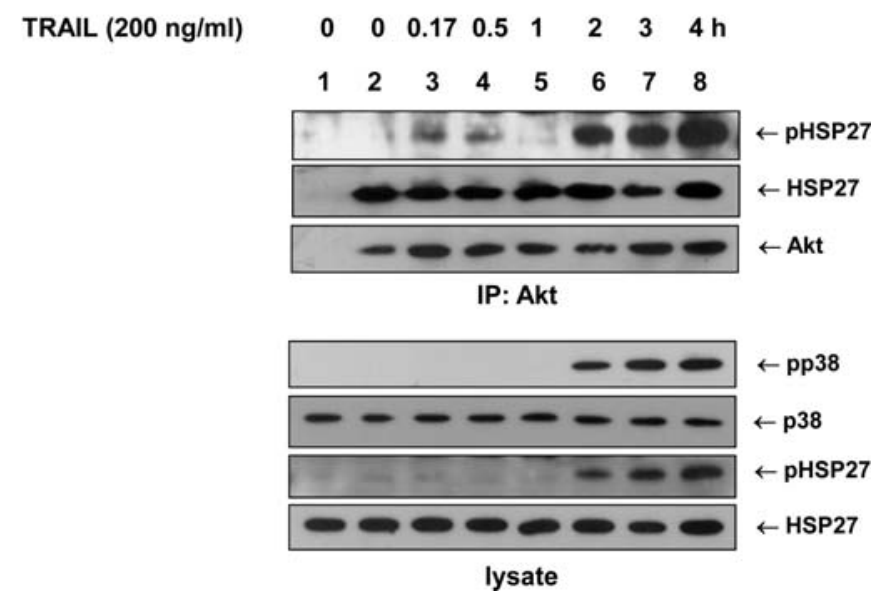

C

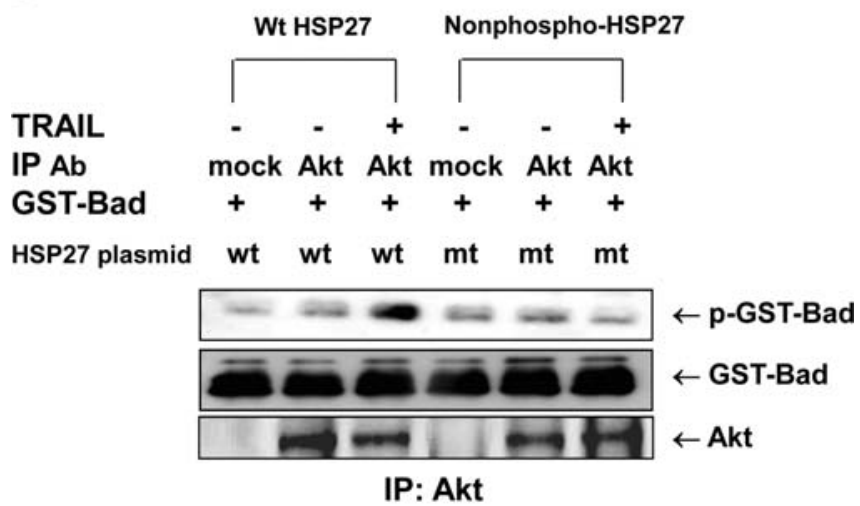

Figure 4. Akt catalytic activity dependence on the phospho-p38/phosphoHSP27 signaling pathway. (A) DU-145 cells pretreated with or without p38 inhibitor were lysed after TRAIL treatment, and the lysates were immunoprecipitated with anti-Akt antibody. Akt catalytic activity was examined in vitro using GST-Bad protein as a substrate. GST-Bad and phosphorylated GST-Bad were detected with anti-Bad and anti-phospho-Ser-136-Bad antibodies, respectively. Immunoprecipitated Akt was detected with rabbit anti-Akt antibody (upper panel). Cell lysates were immunoblotted with antiphospho-p38, anti-p38, anti-phospho-HSP27, anti-HSP27, anti-phospho-Akt or anti-actin antibody, respectively (lower panel). Phospho-HSP27 served as a positive control for p38 inhibitor treatment. (B) Cell lysates were immunoprecipitated with anti-Akt antibody. Phosphorylated HSP27, HSP27, or Akt was detected with anti-phospho-HSP27, anti-HSP27, or anti-Akt antibody, respectively (upper panel). Cell lysates were immunoblotted with antiphospho-p38, anti-p38, anti-phospho-HSP27, or anti-HSP27, respectively (lower panel). (C) Cells were transfected with pcDNA3.1+/HSP27 (wildtype) or mutant-type (3A HSP27). After a 48-h incubation, cells were treated with TRAIL (200 ng/ml) for $2 \mathrm{~h}$ and then immunoprecipitated with anti-Akt antibody. Akt catalytic activity was examined in vitro using GST-Bad protein as a substrate. GST-Bad and phosphorylated GST-Bad were detected with anti-Bad and anti-phospho-Ser-136-Bad antibodies, respectively. Immunoprecipitated Akt was detected with rabbit anti-Akt antibody (upper panel). Cell lysates were immunoblotted with anti-phospho-HSP27, antiHSP27, anti-PARP, or anti-actin antibodies (lower panel). wt, wild-type; mt, mutant-type

catalytic inhibitor via competitive binding in the ATP pocket, phosphorylation of p38 in the presence of inhibitor did not change substantially.

Phosphorylation of HSP27 is necessary for TRAIL-induced Akt catalytic activation. A remaining question was the biological role of phosphorylated HSP27 during TRAIL treatment. We observed that Akt interacted with HSP27, and that phosphorylated HSP27 showed increased interaction with Akt during TRAIL treatment (Fig. 4B). To elucidate the effect of phosphorylated HSP27 on Akt catalytic activity, Akt catalytic activity was assayed after overexpression of wild-type HSP27 and a non-phosphorylatable 3A-HSP27 mutant, kindly provided by Dr Michael J. Welsh (University of Michigan). TRAIL-induced Akt catalytic activity was significantly repres-sed in cells that overexpressed the nonphosphorylatable 3A-HSP27 mutant (Fig. 4C), suggesting that phosphorylated HSP27 is required for TRAIL-induced Akt catalytic activation. found complete repression of HSP27 phosphorylation in the presence of SB203580. Since SB203580 acts as a p38 
A

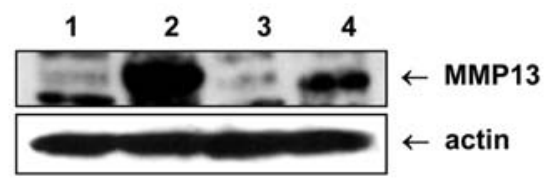

B

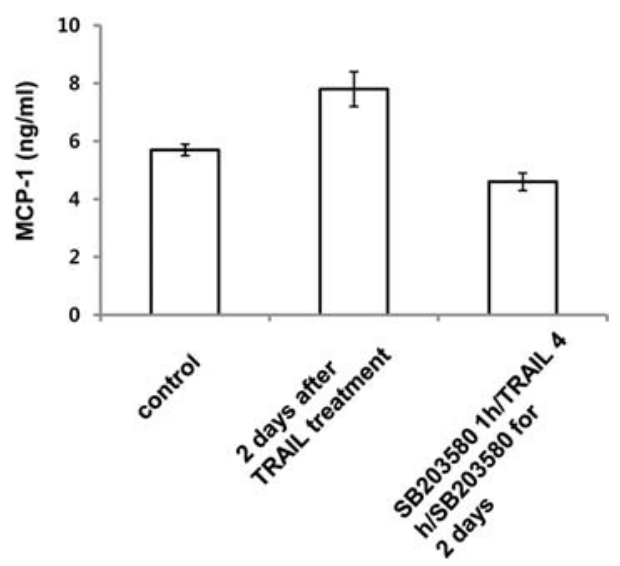

C

control
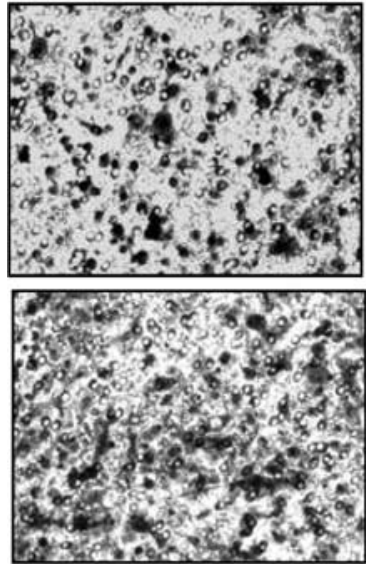

SB203580 for 2 days
2 days after TRAIL
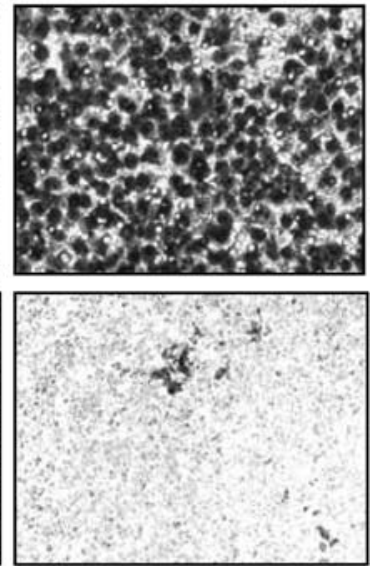

SB203580 $1 \mathrm{~h}$ /TRAIL 4 h/SB203580 for 2 days

Figure 5. Biological role of $\mathrm{p} 38$ during TRAIL-induced invasive activity. (A) The level of MMP13 was examined using the p38 inhibitor SB203580 (20 $\mu$ M). Following $1 \mathrm{~h}$ of pretreatment with SB203580, cells were treated with TRAIL $(200 \mathrm{ng} / \mathrm{ml})$ for $4 \mathrm{~h}$. After TRAIL treatment, the remaining cells were incubated with SB203580 for two days. (Lane 1, control; lane 2, attached cells after two days of TRAIL treatment; lane 3, SB203580; lane 4, SB203580 pretreated with TRAIL treatment followed by SB203580 for two days.) (B) Following $1 \mathrm{~h}$ pretreatment with SB203580, cells were treated with TRAIL (200 ng/ml) for $4 \mathrm{~h}$. After TRAIL treatment, the remaining cells were incubated with SB203580 for two days. The supernatants from attached cells were harvested two days after TRAIL treatment, and the levels of MCP-1 were quantified using a commercially available sandwich-type ELISA (R\&D Systems). (C) DU-145 cells were cultured in invasion chambers after TRAIL treatment $(200 \mathrm{ng} / \mathrm{ml}$ for $4 \mathrm{~h})$ with or without SB203580 $(20 \mu \mathrm{M})$ pretreatment. The remaining DU-145 cells in chambers with a thin layer of ECMatrix were cultured in serum-free media with or without SB203580 for two days. Invasive cells that migrated through the ECM layer and adhered to the bottom of the polycarbonate membrane in $10 \%$ serum-containing media were stained.

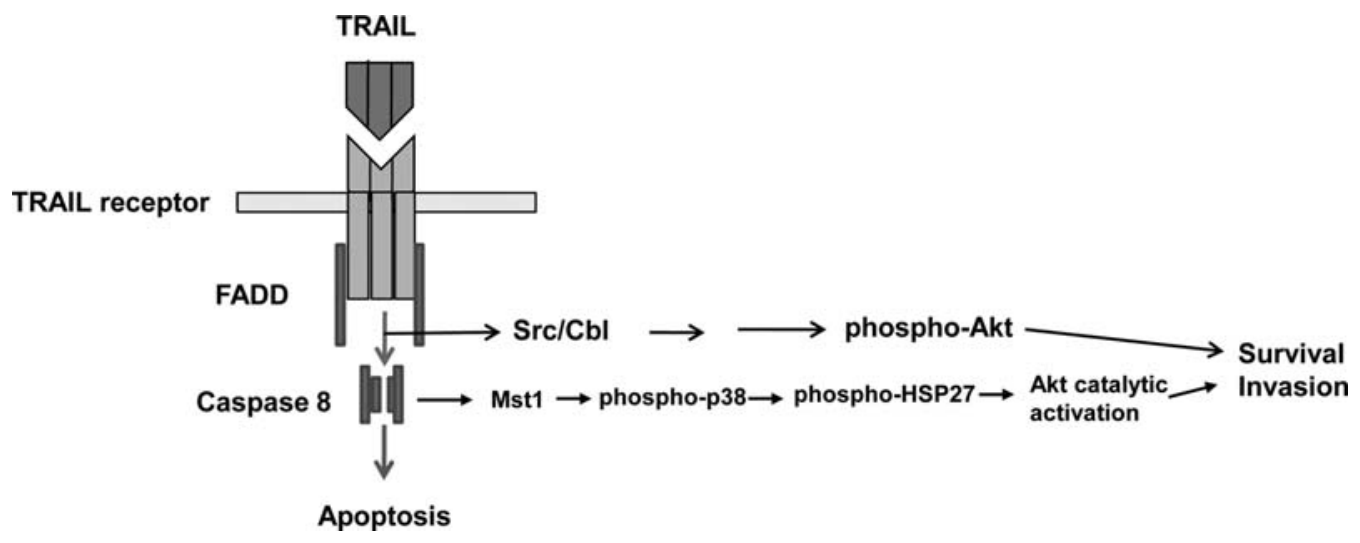

Figure 6. Schematic diagram of TRAIL-induced apoptosis and anti-apoptosis signaling pathways.

TRAIL-induced invasive activity is dependent on the TRAILp38 signaling pathway. Varfolomeev et al (32) suggested that TRAIL-dependent activation of the kinase pathway is associated with increased production of the chemokine MCP-1 that plays a role in prostate cancer invasion and metastasis $(14,32)$. Based on our finding of a TRAIL-dependent increase in Akt catalytic activity that was mediated by phosphorylated HSP27 and p38 activations (Fig. 4A and C), we therefore decided to use p38 inhibitor (SB203580) to investigate the involvement of p38 in cell invasion activity, both during and after TRAIL treatment. Overexpression of MMP13 or MCP-1 was repressed in the presence of p38 inhibitor before and after acquisition of TRAIL resistance (Fig. 5A and B), suggesting that $\mathrm{p} 38$ activation was transmitted to its potential downstream signals such as Akt, MMP13 or MCP-1 to produce acquired TRAIL resistance and TRAIL-induced invasion. We used ECMatrix to confirm that the acquired cells had increased invasive potential, and that p38 activation was imperative for this TRAIL-induced invasive activity (Fig. 5C). DU-145 cells with acquired TRAIL resistance that were cultured in invasion chambers with a thin layer of ECMatrix displayed much higher invasive activity than did 
p38 inhibitor-pretreated DU-145 cells with acquired TRAIL resistance.

Working hypothesis of anti-apoptotic role of p38 induced by TRAIL. Collectively, our previous and current experimental data suggest that TRAIL induces both anti-apoptotic and apoptotic signals in parallel. When TRAIL binds to its receptors, it activates caspase 8 through adaptors such as FADD and eventually leads to apoptosis. At the same time or subsequently, TRAIL also induces Akt phosphorylation through Src/c-Cbl and/or Akt catalytic activation through p38 activation/phosho-HSP27, respectively, resulting in survival or invasive activation (Fig. 6).

\section{Discussion}

We have previously investigated the side-effects of acquired TRAIL resistance during TRAIL-induced apoptosis. These studies showed that phosphorylation of Akt and rapid degradation of TRAIL receptors (DR4/5) during TRAIL treatment were the underlying mechanisms of acquired TRAIL-resistance $(7,15,33)$. However, as reported elsewhere (17), MAPKs are also activated during TRAIL-induced apoptosis, and their activation is caspase 8-dependent. Here we reconciled these findings by investigating the biological function of p38 activation induced by TRAIL treatment in the prostate cancer cell line, DU-145.

As shown in our previous study (16), phosphorylation of Akt does not always reflect its own catalytic activity. Our current study further reinforced the hypothesis that Akt activity is mainly derived from Akt catalytic activity rather than Akt phosphorylation (Figs. 1C and 2B). In addition, the increase in TRAIL-induced Akt catalytic activity in TRAILsensitive cells, but not in TRAIL-resistant cells, implied that cancer cells possess a mechanism to protect themselves from cell death in a negative feedback manner. This finding suggested that TRAIL-mediated activation of caspase 8 and its downstream signals such as p38 could play an essential role in promoting Akt catalytic activation. As noted by Fan and Bergmann (34), maintenance of cell homeostasis is a crucial compensatory mechanism for the survival of multicellular organisms against cell death.

The serine/threonine kinase Akt regulates multiple biological processes, including survival, proliferation, cell growth and glycogen metabolism (35-37). A number of pro-apoptotic proteins have been identified as direct Akt substrates, including Forkhead transcription factors, caspase 9 and Bad (38-43). Phosphorylation by Akt suppresses the pro-apoptotic functions of these molecules. How Akt can mediate upstream signaling to its appropriate downstream signals without impairing other cellular functions in the presence of so many substrates with different cellular functions remains an intriguing question. Fig. 2A displays one possible answer to this question. TRAIL induced Akt activation only when Bad protein was used as a substrate and not when GSK-3 protein was used as a substrate. These results provide a possible mechanism by which a single Akt molecule can finely regulate various signals depending on the nature of the stimulation. The Akt binding pocket for Bad, but not that for GSK-3, may be specifically affected by TRAIL treatment in a positive manner.
The main biological function of phosphorylated HSP27 during TRAIL treatment was Akt activation, which in turn resulted in increased invasive activities of cancer cells. However, as previously reported, phosphorylated HSP27 can induce down-regulation of chaperone action and resistance against oxidative stress. A plausible explanation for this discrepancy can be found in several studies which have shown that MAPKs such as JNK, p38 or ERK1/2 may play dual roles depending on the duration and magnitude of their action $(21,44)$. For example, p38 has various diverse effects depending on stimuli, cellular context, or even cell type $(45,46)$. In other words, in certain conditions, p38 functions as a suppressor of cell proliferation and tumorigenesis (47), while in other cases, p38 regulates invasion and growth of head and neck squamous carcinoma cells (48) or protects against TNF- $\alpha$-provoked apoptosis in prostate cancer cells or murine fibroblasts $(49,50)$. Phosphorylated HSP27 may also play different roles depending on the duration and magnitude of its action, a possibility that is currently being investigated.

\section{Acknowledgements}

This work was supported by the Basic Science Research Program through the National Research Foundation of Korea (NRF) funded by the Ministry of Education, Science and Technology (2009-0071809, 2010-0007689; J.J. Song), Yonsei University Research Fund (2009-0014; J.J. Song) and by a faculty research grant of Yonsei University College of Medicine (2009-0113, 2010-0081; J.J. Song). This study was also supported by the Industrial Strategic technology and development program, 10035562, Development of nucleic acid-based anti-cancer drugs overcoming the immunotherapy resistance, funded by the Ministry of Knowledge Economy (MKE, Korea). So Young Kim and Seeun Oh are supported by the Brain Korea 21 project for Medical Science, Yonsei University, College of Medicine, Seoul, Republic of Korea. This study was also supported by NCI grant fund (CA140554; Y.J. Lee).

\section{References}

1. Zhu H, Zhang L, Huang X, Davis JJ, Jacob DA, Teraishi F, Chiao P and Fang B: Overcoming acquired resistance to TRAIL by chemotherapeutic agents and calpain inhibitor I through distinct mechanisms. Mol Ther 9: 666-673, 2004.

2. Zhang L and Fang B: Mechanisms of resistance to TRAILinduced apoptosis in cancer. Cancer Gene Ther 12: 228-237, 2005.

3. Secchiero P, Zerbinati C, Rimondi E, Corallini F, Milani D, Grill V, Forti G, Capitani S and Zauli G: TRAIL promotes the survival, migration and proliferation of vascular smooth muscle cells. Cell Mol Life Sci 61: 1965-1974, 2004.

4. Morel J, Audo R, Hahne M and Combe B: Tumor necrosis factor-related apoptosis-inducing ligand (TRAIL) induces rheumatoid arthritis synovial fibroblast proliferation through mitogen-activated protein kinases and phosphatidylinositol 3-kinase/Akt. J Biol Chem 280: 15709-15718, 2005.

5. Zauli G, Sancilio S, Cataldi A, Sabatini N, Bosco D and Di Pietro R: PI-3K/Akt and NF-kappaB/IkappaBalpha pathways are activated in Jurkat T cells in response to TRAIL treatment. J Cell Physiol 202: 900-911, 2005.

6. Trauzold A, Siegmund D, Schniewind B, Sipos B, Egberts J, Zorenkov D, Emme D, Röder C, Kalthoff $\mathrm{H}$ and Wajant $\mathrm{H}$ : TRAIL promotes metastasis of human pancreatic ductal adenocarcinoma. Oncogene 25: 7434-7439, 2006.

7. Song JJ, An JY, Kwon YT and Lee YJ: Evidence for two modes of development of acquired TRAIL resistance: Involvement of Bcl-xL. J Biol Chem 282: 319-328, 2007. 
8. Brinckerhoff CE, Rutter JL and Benbow U: Interstitial collagenases as markers of tumor progression. Clin Cancer Res 6: 4823-4830, 2000.

9. Freije JM, Díez-Itza I, Balbín M, Sánchez LM, Blasco R, Tolivia J and López-Otín C: Molecular cloning and expression of collagenase-3, a novel human matrix metalloproteinase produced by breast carcinomas. J Biol Chem 269: 16766-16773, 1994.

10. Johansson N, Airola K, Grénman R, Kariniemi AL, SaarialhoKere U and Kähäri VM: Expression of collagenase-3 (matrix metalloproteinase-13) in squamous cell carcinomas of the head and neck. Am J Pathol 151: 499-508, 1997.

11. Uría JA, Balbín M, López JM, Alvarez J, Vizoso F, Takigawa M and López-Otín C: Collagenase-3 (MMP-13) expression in squamous cell carcinomas of the head and neck. Am J Pathol 153: 91-101, 1998 .

12. Bostrom PJ, Ravanti L, Reunanen N, Aaltonen V, Söderström KO, Kähäri VM and Laato M: Expression of collagenase-3 (matrix metalloproteinase-13) in transitional-cell carcinoma of the urinary bladder. Int J Cancer 88: 417-423, 2000.

13. Lu Y, Xial G, Galson DL, Nishio Y, Mizokami A, Keller ET, Yao Z and Zhang J: PTHrP-induced MCP-1 production by human bone marrow endothelial cells and osteoblasts promotes osteoclast differentiation and prostate cancer cell proliferation and invasion in vitro. Int J Cancer 121: 724-733, 2007.

14. Lu Y, Cai Z, Galson DL, Xiao G, Liu Y, George DE, Melhem MF, Yao Z and Zhang J: Monocyte chemotactic protein-1 (MCP-1) acts as a paracrine and autocrine factor for prostate cancer growth and invasion. Prostate 66: 1311-1318, 2006.

15. Song JJ, Kim JH, Sun BK, Alcala Jr MA, Bartlett DL and Lee YJ: $\mathrm{c}-\mathrm{Cbl}$ acts as a mediator of Src-induced activation of the PI3KAkt signal transduction pathway during TRAIL treatment. Cell Signal 22: 377-385, 2010 .

16. Song JJ and Lee YJ: Dissociation of Akt1 from its negative regulator JIP1 is mediated through the ASK1-MEK-JNK signal transduction pathway during metabolic oxidative stress: a negative feedback loop. J Cell Biol 170: 61-72, 2005.

17. Song JJ and Lee YJ: Differential cleavage of Mst1 by caspase-3/-7 is responsible for TRAIL-induced activation of the MAPK superfamily. Cell Signal 20: 892-906, 2008.

18. Leppä S and Bohmann D: Diverse functions of JNK signaling and c-Jun in stress response and apoptosis. Oncogene 18: 6158-6162, 1999.

19. Matsuzawa A and Ichijo H: Molecular mechanisms of the decision between life and death: regulation of apoptosis by apoptosis signal-regulating kinase 1. J Biochem 130: 1-8, 2001

20. Liu J and Lin A: Role of JNK activation in apoptosis: a doubleedged sword. Cell Res 15: 36-42, 2005.

21. Roulston A, Reinhard C, Amiri P and Williams LT: Early activation of c-Jun N-terminal kinase and p38 kinase regulate cell survival in response to tumor necrosis factor alpha. J Biol Chem 273: 10232-10239, 1998.

22. Mansouri A, Ridgway LD, Korapati AL, Zhang Q, Tian L, Wang Y, Siddik ZH, Mills GB and Claret FX: Sustained activation of $\mathrm{JNK} / \mathrm{p} 38$ MAPK pathways in response to cisplatin leads to Fas ligand induction and cell death in ovarian carcinoma cells. J Biol Chem 278: 19245-19256, 2003.

23. Luo Y and DeFranco DB: Opposing roles for ERK1/2 in neuronal oxidative toxicity: distinct mechanisms of ERK $1 / 2$ action at early versus late phases of oxidative stress. J Biol Chem 281: 16436-16442, 2006

24. Zheng C, Lin Z, Zhao ZJ, Yang Y, Niu H and Shen X: MAPKactivated protein kinase-2 (MK2)-mediated formation and phosphorylation-regulated dissociation of the signal complex consisting of p38, MK2, Akt, and Hsp27. J Biol Chem 281: 37215-37226, 2006

25. Rouse J, Cohen P, Trigon S, Morange M, Alonso-Llamazares A, Zamanillo D, Hunt T and Nebreda AR: A novel kinase cascade triggered by stress and heat shock that stimulates MAPKAP kinase-2 and phosphorylation of the small heat shock proteins. Cell 78: 1027-1037, 1994.

26. Garrido C: Size matters: of the small HSP27 and its large oligomers. Cell Death Differ 9: 483-485, 2002.

27. Rogalla T, Ehrnsperger M, Preville X, Kotlyarov A, Lutsch G Ducasse C, Paul C, Wieske M, Arrigo AP, Buchner J and Gaestel M: Regulation of Hsp27 oligomerization, chaperone function, and protective activity against oxidative stress/tumor necrosis factor alpha by phosphorylation. J Biol Chem 274: 18947-18956, 1999
28. Stokoe D, Engel K, Campbell DG, Cohen P and Gaestel M: Identification of MAPKAP kinase 2 as a major enzyme responsible for the phosphorylation of the small mammalian heat shock proteins. FEBS Lett 313: 307-313, 1992.

29. Xu L, Chen S and Bergan RC: MAPKAPK2 and HSP27 are downstream effectors of p38MAP kinase-mediated matrix metalloproteinase type 2 activation and cell invasion in human prostate cancer. Oncogene 25: 2987-2998, 2006.

30. Shankar S, Ganapathy S, Chen Q and Srivastava RK: Curcumin sensitizes TRAIL-resistant xenografts: molecular mechanisms of apoptosis, metastasis and angiogenesis. Mol Cancer 7: 16-28, 2008.

31. Behbakht K, Qamar L, Aldridge CS, Coletta RD, Davidson SA, Thorburn A and Ford HL: Six 1 overexpression in ovarian carcinoma causes resistance to TRAIL-mediated apoptosis and is associated with poor survival. Cancer Res 67: 3036-3042, 2007.

32. Varfolomeev E, Maecker H, Sharp D, Lawrence D, Renz M, Vucic D and Ashkenazi A: Molecular determinants of kinase pathway activation by Apo2 ligand/tumor necrosis factor-related apoptosis-inducing ligand. J Biol Chem 280: 40599-40608, 2005.

33. Song JJ, Szczepanski MJ, Kim SY, Kim JH, An JY, Kwon YT, Alcala Jr MA, Bartlett DL and Lee YJ: c-Cbl-mediated degradation of TRAIL receptors is responsible for the development of the early phase of TRAIL resistance. Cell Signal 22: 553-563, 2010 .

34. Fan Y and Bergmann A: Apoptosis-induced compensatory proliferation. The Cell is dead. Long live the Cell! Trends Cell Biol 18: 467-473, 2008.

35. Franke TF, Kaplan DR and Cantley LC: PI3K: downstream AKTion blocks apoptosis. Cell 88: 435-437, 1997.

36. Vivanco I and Sawyers CL: The phosphatidylinositol 3-kinase AKT pathway in human cancer. Nat Rev Cancer 2: 489-501, 2002.

37. Hajduch E, Litherland GJ and Hundal HS: Protein kinase B (PKB/Akt)-a key regulator of glucose transport? FEBS Lett 492: 199-203, 2001.

38. Brunet A, Bonni A, Zigmond MJ, Lin MZ, Juo P, Hu LS, Anderson MJ, Arden KC, Blenis J and Greenberg ME: Akt promotes cell survival by phosphorylating and inhibiting a Forkhead transcription factor. Cell 96: 857-868, 1999.

39. Cardone MH, Roy N, Stennicke HR, Salvesen GS, Franke TF, Stanbridge E, Frisch S and Reed JC: Regulation of cell death protease caspase-9 by phosphorylation. Science 282: 1318-1321, 1998.

40. Datta SR, Dudek H, Tao X, Masters S, Fu H, Gotoh Y and Greenberg ME: Akt phosphorylation of BAD couples survival signals to the cell-intrinsic death machinery. Cell 91: 231-241, 1997.

41. Hetman M, Cavanaugh JE, Kimelman D and Xia Z: Role of glycogen synthase kinase-3beta in neuronal apoptosis induced by trophic withdrawal. J Neurosci 20: 2567-2574, 2000.

42. Kops GJ, de Ruiter ND, De Vries-Smits AM, Powell DR, Bos JL and Burgering BM: Direct control of the Forkhead transcription factor AFX by protein kinase B. Nature 398: 630-634, 1999.

43. Pap $M$ and Cooper GM: Role of glycogen synthase kinase-3 in the phosphatidylinositol 3-Kinase/Akt cell survival pathway. J Biol Chem 273: 19929-19932, 1998.

44. Zhao Y, Zhao X, Yang B, Neuzil J and Wu K: alpha-Tocopheryl succinate-induced apoptosis in human gastric cancer cells is modulated by ERK1/2 and c-Jun N-terminal kinase in a biphasic manner. Cancer Lett 247: 345-352, 2007.

45. Lüschen S, Scherer G, Ussat S, Ungefroren H and Adam-Klages S: Inhibition of p38 mitogen-activated protein kinase reduces TNF-induced activation of NF-kappaB, elicits caspase activity, and enhances cytotoxicity. Exp Cell Res 293: 196-206, 2004.

46. Bradham C and McClay DR: p38 MAPK in development and cancer. Cell Cycle 5: 824-828, 2006.

47. Wagner EF and Nebreda AR: Signal integration by JNK and p38 MAPK pathways in cancer development. Nat Rev Cancer 9: 537-549, 2009.

48. Junttila MR, Ala-Aho R, Jokilehto T, Peltonen J, Kallajoki M, Grenman R, Jaakkola P, Westermarck J and Kähäri VM: p38alpha and p38delta mitogen-activated protein kinase isoforms regulate invasion and growth of head and neck squamous carcinoma cells. Oncogene 26: 5267-5279, 2007.

49. Ricote M, García-Tuñín I, Fraile B, Fernández C, Aller P, Paniagua R and Royuela M: p38 MAPK protects against TNFalpha-provoked apoptosis in LNCaP prostatic cancer cells. Apoptosis 11: 1969-1975, 2006.

50. Hui L, Bakiri L, Stepniak E and Wagner EF: p38alpha: a suppressor of cell proliferation and tumorigenesis. Cell Cycle 6: 2429-2433, 2007. 